\title{
How Important Are the Standard Measures in Assessing the Clinical Outcomes in Psoriatic Arthritis in Real-world Settings?
}

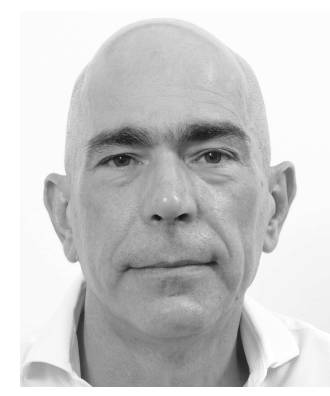

Over the last few years, the worldwide interest in psoriatic arthritis (PsA) has progressively increased because of better comprehension of the pathogenetic pathways that underlie the disease, as well as the ensuing availability on the market of drugs against specific molecular targets. Nevertheless, the selective blocking of a specific target in vivo has provided evidence that the role of a specific pathologic pathway is not absolute and that patients with PsA may benefit from different biological drugs. This suggests that the target to be inhibited varies among patients, and not infrequently within the same patient during the course of the disease. This scenario is consistent with the clinical heterogeneity of PsA and accounts for the compelling need to define clear-cut clinical phenotypes to be treated with the appropriate drug. As a consequence, unlike rheumatoid arthritis (RA), whose therapeutic algorithm is agreed upon all over the world, the best therapeutic strategy to adopt in PsA is still a matter of debate, as the different approaches advocated by the international recommendations from the European League Against Rheumatism and the Group for Research and Assessment of Psoriasis and Psoriatic Arthritis demonstrate ${ }^{1}$. In addition, the complexity of the disease makes PsA disease activity rather difficult to assess: as a result, we still are missing a comprehensive composite score capable of estimating the different clinical manifestations of PsA, and more importantly, the clinical outcomes of our treatments. The latter is maybe the most compelling and critical issue in the field of PsA together with the still-open debate about which measures should be carried out in selecting the eligible patients to commence a biologic drug and in evaluating the clinical outcomes.

This matter is definitely far more troublesome in real-world settings. However, are we really sure that this is the crux of the matter? Are we persuaded that we do need validated tools to assess the clinical response in patients with PsA in the real world? We must never forget that unselected patients with PsA in routine clinical practice are certainly different from those screened and enrolled in randomized clinical trials $(\mathrm{RCT})$. In the latter, we are committed to demonstrate the efficacy of an active drug over placebo, and therefore the patient's cohort must be homogeneous and without potential confounding factors such as comorbidities, severe obesity, or infective risk. In the former, on the other hand, we need to have reliable and feasible tools to evaluate the clinical outcomes, and most importantly, to predict the clinical response. In the current issue of The Journal, Ogdie and colleagues ${ }^{2}$ have assessed the 12-month clinical response to tumor necrosis factor inhibitors (TNFi) in a large cohort of patients with PsA from the North American Corrona registry, using the Clinical Disease Activity Index (CDAI) as a measure of clinical outcomes. CDAI is a handy and reliable composite index based on patient-reported global assessment (PtGA), physician's global assessment, and 28 tender and 28 swollen joint counts $^{3}$. However, it must be said that CDAI is neither validated for PsA nor applied in RCT because skin, enthesis, distal interphalangeal joints, and dactylitis are not taken into account. The authors provided evidence that $14 \%$ of patients with PsA achieved CDAI-based remission and 37\% reached low disease activity (LDA) after 1 year of TNFi therapy.

This is not the first study carried out on PsA patients' data recorded in the Corrona registry and based on CDAI. Indeed, in a study by Huynh, et $a l^{4}$, patients with PsA $(\mathrm{n}=302)$ discontinuing TFNi while experiencing LDA defined by $\mathrm{CDAI} \leq 10$ were followed up, and at last clinic visit, $55.1 \%$ $(n=179)$ of them were still in LDA without TNFi therapy. A simple explanation is that CDAI is a quick and valuable surrogate score to measure disease activity in RA patients, with the advantage of being computed also when laboratory investigations are lacking. CDAI may be the most common tool used by rheumatologists in North America to assess disease activity in all patients with polyarthritis in real-life settings; this fact is used to justify attempts to extend its use to PsA, although CDAI has not been validated for PsA. More recently ${ }^{5}$, a retrospective study of patients with PsA across 10 years from southern Norway was carried out to assess the proportion of patients achieving the state of disease

See Predictors of remission in PsA with TNFi, page 475

Personal non-commercial use only. The Journal of Rheumatology Copyright @ 2019. All rights reserved. 
remission estimated according to different clinical composite indices, including CDAI and the specific Disease Activity Index for Psoriatic Arthritis (DAPSA). In the last 5 years, the ratio between patients in remission based on CDAI and those based on DAPSA did not substantially change over time, suggesting that unspecific clinical scores might also be helpful in clinical practice to identify the achievement of a clinical outcome. However, CDAI as it is cannot cover the whole spectrum of clinical manifestations of PsA, and its application is likely to cause a selection bias and to discard PsA patients with oligoarticular phenotype, just to mention one. Indeed, in the paper by $\mathrm{Ogdie}^{2}$, only patients having at least 3 tender or swollen joints were included into the analysis; only 883 out of 1832 TNFi initiations met this criterion. Arguably, patients with fewer than 3 joints involved should have a very low CDAI at baseline. This implies that a proportion of PsA patients, with active disease according to the physician, are initiated to TNFi with a low CDAI at baseline. They are probably patients with oligoarticular and/or axial involvement. On the other hand, the self-reported PtGA computed into the CDAI can reflect the global effectiveness of the treatment, and therefore the measurement of disease activity based on CDAI can be deemed trustworthy, but still in a selected cohort of patients with PsA.

The reliability of CDAI in this study ${ }^{2}$ can be indirectly confirmed by the findings that, as previously reported in other PsA cohorts, female sex and comorbidities were negatively associated to the achievement of the clinical outcome. As expected, in female patients the HR of reaching CDAI-based remission was 0.62 (95\% CI $0.40-0.97)$. In addition to this, obesity (HR 0.51, 95\% CI 0.32-0.81) and hypertension (HR $0.55,95 \%$ CI $0.32-0.95$ ) reduced the chances of achieving disease remission. These results give us the opportunity to briefly speculate on the emerging topic of comorbidities. Once again, high body mass proves to decrease the effectiveness of the classical TNFi in PsA. Actually, there are some discrepancies in the literature; a few studies had not fully confirmed obesity as a negative predictor of good clinical response ${ }^{6}$. This may be because obesity is often clustered with other comorbidities that can bias the clinical effect of obesity itself. This would mean that the effect of obesity should be addressed in the wider field of comorbidities, instead of being considered alone. In this regard, the modified Rheumatic Disease Comorbidity Index is a comprehensive score of comorbidities, including obesity, diabetes, major cardiovascular events, hypertension, and others. It has been found to limit the achievement of 28-joint Disease Activity Score-driven remission in 208 patients with PsA (HR $0.77,95 \%$ CI 0.61-0.97), along with female sex (HR $0.67,95 \%$ CI $0.30-0.85)^{7}$. A further demanding matter that needs to be considered, especially in PsA, is comorbid fibromyalgia (FM). FM can mislead the assessment of disease activity of PsA by interfering with the detection of entheseal points and the patient's perception of pain and global disease ${ }^{8}$. Additionally, FM seems to be a strong negative predictor of the achievement of 12-month minimal disease activity as well as DAPSA-based remission in 238 biologic-naive patients with PsA taking TNFi (unsubmitted personal data).

PsA is an extremely complex disease, and when dealing

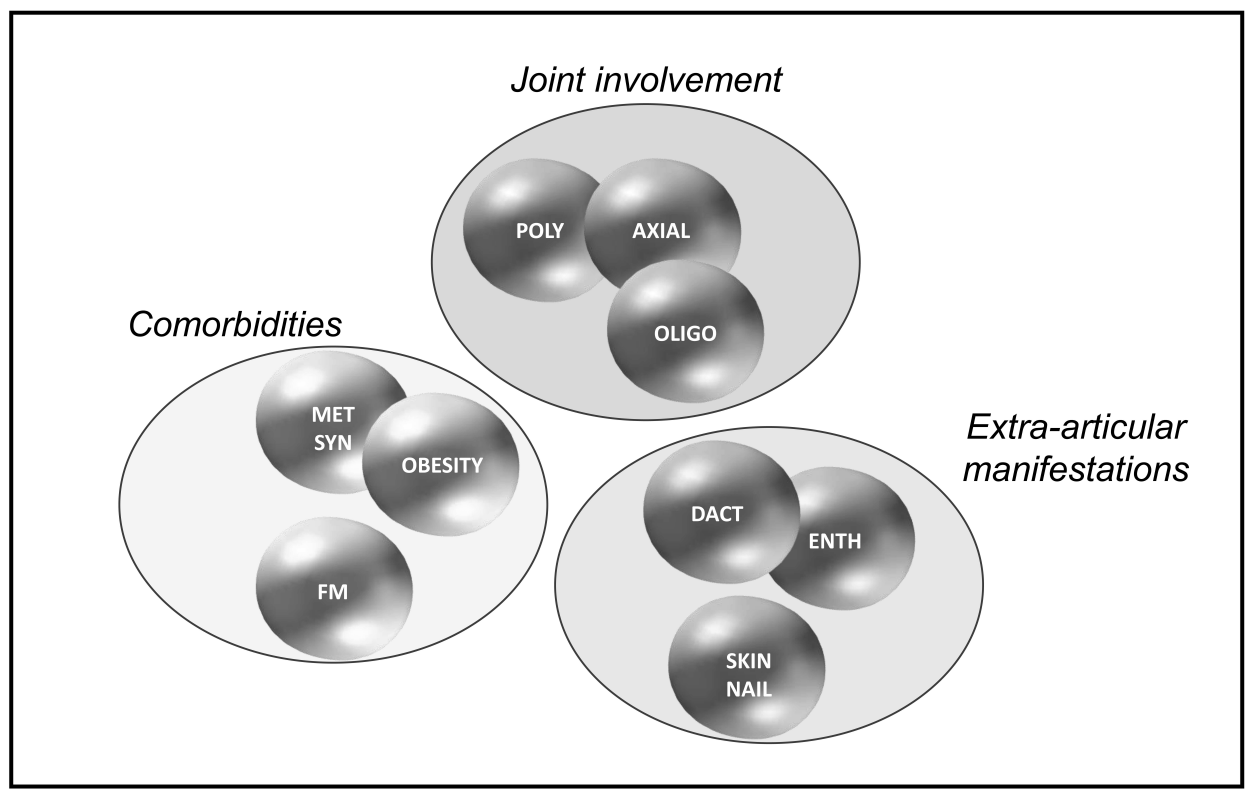

Figure 1. Psoriatic arthritis (PsA) spectrum. Each large domain encompasses a cluster of single items with a different extent of overlap. The ideal approach to PsA should be based on a global perspective. POLY: polyarticular; OLIGO: oligoarticular; MET SYN: metabolic syndrome; FM: fibromyalgia; DACT: Dactylitis; ENTH: Enthesitis.

Personal non-commercial use only. The Journal of Rheumatology Copyright @ 2019 . All rights reserved. 
with patients we must be aware of at least 3 large clinical domains (Figure 1): articular, extraarticular, and comorbidities, each of which embraces in its turn a cluster of items with a different extent of overlap. A comprehensive evaluation of PsA as a whole in real-world settings seems difficult to carry out. However, adopting a specific set of measures of clinical outcomes, even though they are not validated, can be rather useful, providing that the results are not generalized to all PsA populations but are limited to the PsA subset selected in the analysis. This approach, although incomplete and imperfect, can be of some aid in current clinical practice.

\section{FLORENZO IANNONE (D), MD, $\mathrm{PhD}$,}

Associate Professor of Rheumatology,

Department of Emergence and Organ Transplantations, Rheumatology Unit,

Università degli Studi di Bari Aldo Moro, Bari, Italy.

Address correspondence to Dr. F. Iannone, Rheumatology Unit, Policlinico, Piazza G. Cesare 11, 70124 Bari, Italy.

E-mail: florenzo.iannone@uniba.it

\section{REFERENCES}

1. Gossec L, Coates LC, de Wit M, Kavanaugh A, Ramiro S, Mease PJ, et al. Management of psoriatic arthritis in 2016: a comparison of EULAR and GRAPPA recommendations. Nat Rev Rheumatol 2016;12:743-50.

2. Ogdie A, Palmer J, Greenberg J, Curtis J, Harrold L, Kavanaugh A, et al. Predictors of achieving remission among patients with psoriatic arthritis initiating a TNF inhibitor. J Rheumatol 2019;46:475-82.

3. Aletaha D, Smolen J. The Simplified Disease Activity Index (SDAI) and the Clinical Disease Activity Index (CDAI): a review of their usefulness and validity in rheumatoid arthritis. Clin Exp Rheumatol 2005;23:S100-8.

4. Huynh DH, Boyd TA, Etzel CJ, Cox V, Kremer J, Mease P, et al. Persistence of low disease activity after tumour necrosis factor inhibitor (TNFi) discontinuation in patients with psoriatic arthritis. RMD Open 2017;3:e000395.

5. Haugeberg G, Michelsen B, Tengesdal S, Hansen IJW, Diamantopoulos A, Kavanaugh A. Ten years of follow-up data in psoriatic arthritis: results based on standardized monitoring of patients in an ordinary outpatient clinic in southern Norway. Arthritis Res Ther 2018;20:160.

6. Lupoli R, Pizzicato P, Scalera A, Ambrosino P, Amato M, Peluso R, et al. Impact of body weight on the achievement of minimal disease activity in patients with rheumatic diseases: a systematic review and meta-analysis. Arthritis Res Ther 2016;18:297.

7. Iannone F, Salaffi F, Fornaro M, Di Carlo M, Gentileschi S, Cantarini L, et al. Influence of baseline modified rheumatic Disease Comorbidity Index (mRDCI) on drug survival and effectiveness of biological treatment in patients affected with Rheumatoid Arthritis, Spondyloarthritis, and Psoriatic Arthritis in real-world settings. Eur J Clin Invest 2018:e13013.

8. Marchesoni A, De Marco G, Merashli M, McKenna F, Tinazzi I, Marzo-Ortega $\mathrm{H}$, et al. The problem in differentiation between psoriatic-related polyenthesitis and fibromyalgia. Rheumatology 2018;57:32-40.

J Rheumatol 2019;46:450-2; doi:10.3899/jrheum.180906 